\title{
Rotating superfluids and spinning charged operators in conformal field theory
}

\author{
Gabriel Cuomo, ${ }^{1}$ Anton de la Fuente, ${ }^{1}$ Alexander Monin, ${ }^{2,3}$ David Pirtskhalava, ${ }^{1,4}$ and Riccardo Rattazzi ${ }^{1}$ \\ ${ }^{1}$ Theoretical Particle Physics Laboratory, Institute of Physics, EPFL, 1015 Lausanne, Switzerland \\ ${ }^{2}$ Laboratory of Particle Physics and Cosmology, Institute of Physics, EPFL, 1015 Lausanne, Switzerland \\ ${ }^{3}$ Department of Theoretical Physics, University of Geneva, 1205 Geneva, Switzerland \\ ${ }^{4}$ Theoretical Physics Group, Blackett Laboratory, Imperial College, London SW7 2AZ, United Kingdom
}

(Received 13 November 2017; published 15 February 2018)

\begin{abstract}
We compute the scaling dimensions of operators with large global charge and spin in $2+1$ dimensional conformal field theories. By the state-operator correspondence, these operators correspond to superfluids with vortices and can be systematically studied using effective field theory. As the spin increases from zero to the unitarity bound, the superfluid state corresponding to the lowest dimension operator passes through three distinct regimes: (i) a single phonon, (ii) two vortices, and (iii) multiple vortices. We also compute correlation functions with two such operators and the Noether current.
\end{abstract}

DOI: 10.1103/PhysRevD.97.045012

\section{INTRODUCTION}

Perhaps a way to phrase the main difference between high energy physics and condensed matter physics is that high energy physics mostly occurs in the vacuum while condensed matter physics occurs at finite density. In a conformal field theory (CFT), this particular difference disappears: The state-operator correspondence maps finite density states to local operators and therefore maps finite density correlators to vacuum correlators.

Recently, this idea was applied using superfluids [1-9]. Superfluids are finite density states and correspond to operators with large global charge. Furthermore, superfluids are described by an effective field theory (EFT), allowing the computation of correlators in a systematic perturbative expansion [10]. This EFT was used to study the CFT operator spectrum at large global charge [1-9].

In an independent line of research, much work was devoted to using the conformal bootstrap $[11,12]$ to study the CFT operator spectrum at large spin [13-24]. This motivated us to ask the following question: Can large spin operators also be studied using EFT techniques? An obvious approach to this question is to start with the large charge operators studied by Refs. [1-9] and then proceed by adding increasing amounts of spin to them. This translates to adding angular momentum to the corresponding superfluid.

Published by the American Physical Society under the terms of the Creative Commons Attribution 4.0 International license. Further distribution of this work must maintain attribution to the author(s) and the published article's title, journal citation, and DOI. Funded by SCOAP ${ }^{3}$.
Experimentally, when angular momentum is added to a superfluid in the laboratory, vortices develop [25]. However, the superfluid EFT used by Refs. [1-9] does not incorporate vortices; all angular momentum is carried by phonons alone. This suggests that the EFT would incorrectly describe high angular momentum states. Conveniently, a superfluid EFT that incorporates vortices was recently constructed [26]. We will use this EFT to study operators that have large spin as well as large charge.

\section{RESULTS}

We found that we can study large charge operators with arbitrary spin, provided that the spin is parametrically below the unitarity bound. This range of spins is disjoint from the range in which the large spin bootstrap work [13-24] is valid - that work only applies to operators parametrically close to the unitarity bound. Therefore, our results complement the bootstrap results.

We calculated the dimension $\Delta$ of the lowest dimension operator with charge $Q \gg 1$ and spin $J$. As $J$ varies from 0 to $\Delta$, the corresponding superfluid state passes through three qualitatively distinct regimes. We will simply state the results now and derive them later. The results are displayed at leading order in both large $Q$ and large $J$. At this order, there is only a single free parameter $\alpha$ for the entire range of $J$.

For $0 \leq J \lesssim \sqrt{Q}$, the lowest energy state has no vortices and consists of a single phonon of angular momentum $J$. The corresponding operator dimension $\Delta$ is $[1,3]$

$$
\Delta=\alpha Q^{3 / 2}+\frac{J}{\sqrt{2}} .
$$

For $\sqrt{Q} \lesssim J \leq Q$, the lowest energy state consists of a vortex-antivortex pair whose separation increases with $J$. The corresponding operator dimension $\Delta$ is 


$$
\Delta=\alpha Q^{3 / 2}+\frac{\sqrt{Q}}{3 \alpha} \ln \frac{J}{\sqrt{Q}} .
$$

For $Q<J \lesssim Q^{3 / 2}$, the lowest energy state consists of multiple vortex-antivortex pairs distributed so that the superfluid has the same velocity profile as that of a rotating rigid body [27]. The corresponding operator dimension $\Delta$ is

$$
\Delta=\alpha Q^{3 / 2}+\frac{1}{2 \alpha} \frac{J^{2}}{Q^{3 / 2}} .
$$

As $J \rightarrow Q^{3 / 2} \sim \Delta$, the EFT breaks down and, as mentioned, we are unable to reach the spin of the operators studied in Refs. [13-24] by bootstrap methods.

Our results apply to any CFT that satisfies three conditions: First, its large charge sector can be described as a superfluid; second, this superfluid admits vortices; third, the only low energy degrees of freedom are the Goldstone modes of the superfluid. These are the simplest and most natural conditions we can imagine. Because of this, we believe - but cannot prove - that our results apply to a wide range of CFTs with a $U(1)$ global symmetry. For example, we expect that they apply to the critical $O(2)$ model [28] and can be tested in principle. Recently, this question was framed within the conformal bootstrap [29].

\section{GENERAL STRATEGY}

We will now explain in more detail how the above results were derived. Our general strategy consists of combining two powerful tools: EFT and the state-operator correspondence. We begin by considering a $d+1$ dimensional CFT on a cylinder $\mathbb{R} \times S^{d}$. Next, we assume that a given EFT on this cylinder is a valid description of our CFT. Finally, we apply the state-operator correspondence directly to the states of this EFT. Throughout this paper, unless otherwise stated, we shall work at leading order in the derivative and field expansion within EFT [1,3].

Note that this strategy differs from that in Ref. [3], which takes the "top-down" approach of projecting onto a desired state using the Euclidean path integral. Instead, we are taking the "bottom-up" approach of simply assuming an EFT and then quantizing its Hamiltonian while always remaining in Lorentzian spacetime.

As a reminder, in a CFT, there is a one-to-one correspondence between the eigenstates of the Hamiltonian $H$ on $S^{d}$ and the set of scaling operators at any given point. This is called the state-operator correspondence (see $[11,12]$ for reviews). The energy $E$ of a state is related to the scaling dimension $\Delta$ of the corresponding operator by $E=\Delta / R$, where $R$ is the radius of the sphere $S^{d}$. All other conserved quantum numbers of the state (such as global charge and spin) are identical to those of the corresponding operator.

\section{DUAL GAUGE FIELD}

We now specialize the construction of [26] to the cylinder $S^{2} \times \mathbb{R}$. It uses a dynamical gauge field $a_{\mu}$ instead of the more familiar Goldstone field $\pi$. We begin with the effective Lagrangian for a conformal superfluid written in terms of $\pi[1,3,10]$ :

$$
\mathcal{L}=c(\partial \chi)^{3},
$$

where $c$ is an unknown constant and $\chi \equiv \mu t+\pi$. The parameter $\mu$ can be interpreted as the chemical potential. We use the notation $w \equiv\left(g^{\mu \nu} w_{\mu} w_{\nu}\right)^{1 / 2}$, where $g_{\mu \nu}$ is the spacetime metric and $w_{\mu}$ is an arbitrary spacetime vector. We then dualize $\chi$ by formally treating $v_{\mu} \equiv \partial_{\mu} \chi$ as an independent variable and using a Lagrange multiplier $a_{\mu}$ to set the curl of $v_{\mu}$ to zero:

$$
\mathcal{L}=c v^{3}-\frac{1}{2 \pi} a_{\mu} \frac{\epsilon^{\mu \nu \lambda}}{\sqrt{g}} \partial_{\nu} v_{\lambda},
$$

where we use the combination $\epsilon^{\mu \nu \lambda} / \sqrt{g}$ to denote the antisymmetric Levi-Civita tensor. Integrating out $v_{\mu}$ gives

$$
\mathcal{L}=-\kappa f^{3 / 2},
$$

where $f \equiv \sqrt{f_{\mu \nu} f^{\mu \nu}}$ and $f_{\mu \nu} \equiv \partial_{\mu} a_{\nu}-\partial_{\nu} a_{\mu}$. We dropped a boundary term that came from integrating (5) by parts because it is metric independent and thus does not affect the energy momentum tensor. The coefficient $\kappa$ in (6) is related to the coefficient $c$ in (4) as $\kappa=\frac{1}{2^{5 / 4}(3 \pi)^{3 / 2}} \frac{1}{\sqrt{c}}$.

The relation between $\chi$ and $a_{\mu}$ is given by the expression for the $U(1)$ current $j^{\mu}$ :

$$
j^{\mu}=3 c(\partial \chi) \partial^{\mu} \chi=\frac{1}{4 \pi} \frac{\epsilon^{\mu \nu \lambda}}{\sqrt{g}} f_{\nu \lambda} .
$$

In the vacuum, the charge density is $\left\langle j^{0}\right\rangle=\frac{Q}{4 \pi R^{2}}$, where $Q$ is the net charge of the superfluid state and $R$ is the radius of the sphere. This translates to a homogeneous magnetic field

$$
\left\langle f_{\theta \phi}\right\rangle=B \sin \theta \equiv \frac{Q}{2 R^{2}} \sin \theta
$$

and results in a net magnetic flux of $2 \pi Q$ through the sphere. Parametrically, the cutoff $\Lambda$ of our EFT is

$$
\Lambda \sim \sqrt{B} \sim \frac{\sqrt{Q}}{R} .
$$

\section{PARTICLE-VORTEX DUALITY}

Vortices in the superfluid description correspond to heavy charged particles in the gauge theory description. They are treated in a first-quantized form as $0+1$ dimensional worldlines embedded in the $2+1$ dimensional 
spacetime. We will use the terms "vortex" and "charged particle" interchangeably.

To write the effective action for a conformal superfluid with vortices [26], we parametrize the spacetime trajectory of the $p$ th vortex by $X_{p}^{\mu}(\tau)$, where $\tau$ is an auxiliary time parameter. We further impose Weyl and $\tau$-reparametrization invariance, with the former reducing to conformal invariance in the relevant case of a static metric. The action can be organized as a derivative expansion with the lowest order terms given by

$$
\begin{aligned}
S= & -\kappa \int d^{3} x \sqrt{g} f^{3 / 2}-\sum_{p} q_{p} \int a_{\mu} d X_{p}^{\mu} \\
& -\sum_{p} \int d \tau \gamma_{p} \sqrt{f} \sqrt{g_{\mu \nu} X_{p}^{\mu} X_{p}^{\nu}}+\cdots .
\end{aligned}
$$

The first term is the kinetic term (6) for the gauge field. The second term is the leading coupling between a particle of charge $q_{p}$ and the gauge field [30]. The last term can be viewed as the action for a relativistic point particle [30] with mass $\gamma_{p} \sqrt{f}$. The dots in (10) represent terms with at least two derivatives on either $A_{\mu}$ or $X_{p}^{\mu}$, and the coefficient $\gamma_{p}$ should in general be promoted [26] to a function of $j_{\mu} X^{\mu} /(j X)$, where $j_{\mu}$ is the $U(1)$ current (7). However, as we will now explain, the leading order description of our system is fully determined by the first line of (10).

\section{LOWEST LANDAU LEVEL}

Each $X_{p}^{\mu}$ describes the motion of a $2 \mathrm{D}$ particle in a magnetic field and consists of two pairs of canonically conjugate variables. These can be further decomposed into one pair that describes the motion of the guiding center and another pair that describes cyclotron motion. Without interparticle interactions, excitations of the first pair are gapless while excitations of the second pair have a gap $\omega=B / m$, where $m$ is the particle mass. The gapped excitations are the Landau levels [31], and the gaplessness of the guiding center variables is the usual degeneracy of Landau levels.

In our system (10), $\omega \sim \sqrt{B}$, which coincides with the EFT cutoff (9). Thus, within the domain of validity of our EFT, the dynamics of $X^{\mu}$ reduces to that of just the guiding center - the Landau levels are effectively integrated out [32-34]. One well-known fact $[35,36]$ is that the guiding center can be described by dropping the mass term (the second line) from the Lagrangian (10). Physically, this is because in the massless limit, the Landau level gap $\omega \rightarrow \infty$. Formally, this is because the first line in (10) is linear in the particle velocity. This constrains the two physical coordinates to be canonically conjugate to each other, halving the dimension of phase space.

The terms in the second line of (10) are genuine higher derivative corrections. On the one hand, they bring in new states with energy $\sim \sqrt{B}$. On the other, at sufficiently low energy, they can be treated as small perturbations of the leading single derivative term. This is fully analogous to supplementing the 1D Lagrangian $\dot{q}^{2}$ with the four-derivative term $\ddot{q}^{2} / \Lambda^{2}$ : States arise with energy $\sim \Lambda$, but at low energy, the four-derivative term can be treated as a perturbation using standard EFT methods. We leave a systematic study of higher order corrections for future work, though we will briefly mention some effects below.

In what follows, we will therefore derive leading order results by simply dropping the second line in (10) and assume

$$
S=-\kappa \int d^{3} x \sqrt{g} f^{3 / 2}-\sum_{p} q_{p} \int a_{\mu} d X_{p}^{\mu} .
$$

The electrostatic potential coupling will generate nontrivial dynamics and a gap for the guiding centers. As long as particle separations are larger than the cutoff length $1 / \sqrt{B}$, this is within the regime of validity of the EFT.

\section{CLASSICAL ANALYSIS}

We will now compute the classical energy and angular momentum of a state with a given configuration of vortices. By the state-operator correspondence, this gives us the dimension $\Delta$ and spin $J$ of the corresponding operator. We will work to leading order in large $Q$ and in large vortex separations. At this order, the equations of motion from (11) are

$$
\begin{gathered}
\frac{1}{e^{2}} \nabla_{\mu} f^{\mu \nu}=\mathcal{J}^{\nu}, \\
E^{i}=\left(X_{p}\right)_{j} f^{j i},
\end{gathered}
$$

where $E^{i} \equiv f^{i 0}$ is the electric field and $\mathcal{J}^{\nu}$ is the current due to the point charges. The coupling $e^{2}$ is defined as

$$
\frac{1}{e^{2}} \equiv \frac{3 \kappa}{2^{1 / 4}} \frac{1}{\sqrt{B}} .
$$

Equation (12) is Maxwell's equations and (13) imposes that the particles move on trajectories with vanishing Lorentz force. In other words, as expected, the particles exhibit pure drift velocity motion. This is consistent with cyclotron degrees of freedom being integrated out. Thus, the particle velocities are $\left|\vec{X}_{p}\right| \sim|\vec{E}| / B \sim 1 / \sqrt{Q}$ and can be neglected. Our problem has reduced to the $2 \mathrm{D}$ electrostatics of point charges on a sphere in a constant magnetic field.

The stress energy tensor $T_{\mu \nu}=\frac{2}{\sqrt{g}} \frac{\delta S}{\delta g^{\mu \nu}}$ is

$$
T_{\mu \nu}=\frac{\kappa}{\sqrt{f}}\left(-3 f_{\mu \alpha} f_{\nu}{ }^{\alpha}+g_{\mu \nu} f^{2}\right) .
$$


Using this, we calculate the dimension $\Delta$ to be

$$
\Delta=\frac{Q^{3 / 2}}{\sqrt{27 \pi c}}+\frac{R^{3}}{2 e^{2}} \int d \theta d \phi \sin \theta \vec{E}^{2} .
$$

Physically, the first term is the energy stored in the background magnetic field while the second term is the energy stored in the electric field sourced by the particles.

In Coulomb gauge, $E_{i}=\partial_{i} a_{0}$, where $a_{0}$ is the electric potential due to a collection of point charges on a 2 -sphere:

$$
a_{0}(\vec{r})=-\frac{e^{2}}{4 \pi} \sum_{p} q_{p} \ln \left(\vec{r}-\vec{R}_{p}\right)^{2} .
$$

We used embedding coordinates of the 2-sphere in $\mathbb{R}^{3}$, where $\vec{r}=(\sin \theta \cos \phi, \sin \theta \sin \phi, \cos \theta)$ is a unit 3-vector and analogously for $\vec{R}_{p}$. The total electric field energy is a sum of pairwise contributions for each charge:

$$
\frac{R e^{2}}{8 \pi}\left[-\sum_{p \neq r} q_{p} q_{r} \ln \left(\vec{R}_{p}-\vec{R}_{r}\right)^{2}-\sum_{p} q_{p}^{2} \ln 0^{2}\right] .
$$

The last term is the familiar divergent self-energy of a point charge. It will be cut off at angular lengths $\sim 1 / \sqrt{Q}$ (9).

Thus, the dimension $\Delta(16)$ is

$$
\Delta=\alpha Q^{3 / 2}-\frac{\sqrt{Q}}{12 \alpha} \sum_{p \neq r} q_{p} q_{r} \ln \frac{\left(\vec{R}_{p}-\vec{R}_{r}\right)^{2}}{Q},
$$

where $\alpha \equiv 1 / \sqrt{27 \pi c}$, and we used $\sum_{p} q_{p}=0$ to combine the logarithms in (18).

The angular momentum $\vec{J}$ can also be calculated from the stress tensor (15) and is

$$
\vec{J}=-\sum_{p} q_{p} \frac{Q}{2} \vec{R}_{p}
$$

\section{DERIVATION OF RESULTS}

The results stated at the beginning of this paper can now be derived. First, note that the self-energy of a particle of charge $q$ is proportional to $q^{2}$ - this is the second term in (18). Because of this, particles with $|q|>1$ are energetically unfavored.

(i) Equation (1) is derived using the phonon dispersion relation $[1,3], \omega=\left[\frac{1}{2} \ell(\ell+1)\right]^{1 / 2}$, where $\ell$ is angular momentum and $\omega$ is energy. Since $\omega / \ell$ decreases with $\ell$, energy is lowest at fixed $J$ with a single phonon of $\ell=J$.

(ii) Equation (2) is derived by evaluating $\Delta$ (19) and $J$ (20) on a configuration with a single vortexantivortex pair.

(iii) Equation (3) is derived by approximating the vortex distribution as a continuous distribution and then minimizing $\Delta$ (19) for fixed $J$ (20) using variational techniques. This gives a vortex distribution $\rho$ of

$$
\rho=\frac{3}{2 \pi R^{2}} \frac{J}{Q} \cos \theta
$$

and results in the superfluid having the same velocity profile as that of a rigid body [27].

(iv) As $J \rightarrow Q^{3 / 2}$, the electric field $|\vec{E}|$ approaches the magnetic field $B$ and the drift velocities become relativistic. This causes the EFT to break down because the higher order terms neglected in (11) become unsuppressed. The guiding centers becomes as energetic as the cyclotron degrees of freedom and anything else at the EFT cutoff (9).

\section{QUANTIZATION}

Since the vortex positions are continuous, some questions may occur: How many distinct states are there? How does the quantization of angular momentum arise? These questions are answered when we quantize our system of charged particles in a magnetic field. Solving for $a_{0}$ using (17) and ignoring fluctuations of $a_{i}$, our effective Lagrangian (11) becomes

$$
L=\sum_{p} q_{p} \vec{A} \cdot \vec{R}_{p}+\frac{e^{2}}{8 \pi} \sum_{p, r} q_{p} q_{r} \ln \left(\vec{R}_{p}-\vec{R}_{r}\right)^{2},
$$

where $\vec{A}$ is the potential for a magnetic monopole [37,38]. We use the gauge in which $A_{\phi}=\frac{1}{2} Q(1-\cos \theta)$ and $A_{\theta}=0$. This system is known as the "fuzzy sphere" $[39,40]$.

Due to the somewhat complicated form of $\vec{A}$, it is useful to switch to spinor coordinates $[41,42]$ :

$$
\psi \equiv\left(\begin{array}{c}
\cos \frac{\theta}{2} \\
\sin \frac{\theta}{2} e^{i \phi}
\end{array}\right),
$$

where we suppressed the vortex index. In these coordinates, $\vec{R}=\psi^{\dagger} \vec{\sigma} \psi$ and $\vec{A} \cdot \vec{R}=-i Q \psi^{\dagger} \frac{d}{d t} \psi$. This identifies the canonical momentum corresponding to $\psi_{p}$ as $-i Q q_{p} \psi_{p}^{\dagger}$. The canonical commutation relations imply that the angular momentum (20) commutes with the Hamiltonian and satisfies $\left[\mathcal{J}_{i}, \mathcal{J}_{j}\right]=i \epsilon_{i j k} \mathcal{J}_{k}$ [39,40]. We use curly $\overrightarrow{\mathcal{J}}$ to denote the angular momentum operator.

For illustration, consider the case of two vortices of unit charge. The Hamiltonian corresponding to (22) is then

$$
H=\text { const }+\frac{e^{2}}{4 \pi} \ln \overrightarrow{\mathcal{J}}^{2},
$$

where "const" involves terms that are independent of the vortex coordinates, and we used (20) to express $H$ in terms 
of $\overrightarrow{\mathcal{J}}$. The spectrum is thus entirely determined by the spectrum of $\overrightarrow{\mathcal{J}}^{2}$. As is well known, $\overrightarrow{\mathcal{J}}^{2}=J(J+1)$, where $J$ is an integer and for each value of $J$, there are $2 J+1$ degenerate states.

Restoring the constants in (24), the dimension of the corresponding operator is

$$
\Delta=\alpha Q^{3 / 2}+\frac{\sqrt{Q}}{6 \alpha} \ln \frac{J(J+1)}{Q} .
$$

We can trust this equation for $\sqrt{Q} \lesssim J \leq Q$. The lower value is determined by requiring that the vortices be separated by distances larger than the cutoff $\sim 1 / \sqrt{B}$. The upper value occurs when the two particles are at opposite poles.

\section{CORRELATORS}

The EFT can also be used to compute correlation functions [3]. Let us consider correlators involving the $U(1)$ current $j_{\mu}$. From (7) and Gauss's law, we see that the line integral $\oint j_{\mu} d x^{\mu}$ about a closed curve $\mathcal{C}$ at a fixed time is simply $\frac{1}{2 \pi}$ times the total charge $q_{\text {enc }}$ enclosed by $\mathcal{C}$ :

$$
\left\langle\operatorname{vortex}\left|\oint_{\mathcal{C}} j_{\mu} d x^{\mu}\right| \text { vortex }\right\rangle=\frac{e^{2} q_{\mathrm{enc}}}{2 \pi},
$$

where $|\operatorname{vortex}\rangle$ is a generic vortex state. By the stateoperator correspondence, this amounts to a prediction about three-point functions. We will now consider two simple examples.

As a first example, we consider a vortex-antivortex pair located at the north and south poles. Then (26) becomes

$$
\left\langle\operatorname{vortex}\left|j_{\phi}(\theta, \phi)\right| \operatorname{vortex}\right\rangle=\frac{e^{2}}{2 \pi R},
$$

where now $\mid$ vortex $\rangle$ is a state with $J=J_{z}=Q$ and $j_{\phi}$ is the azimuthal component of $j_{\mu}$. In general, the expectation value of a spin-1 operator $j_{\phi}$ in a state $\left|J, J_{z}\right\rangle$ with $J=$ $J_{z}=Q$ is [43]

$$
\left\langle Q, Q\left|j_{\phi}(\theta, \phi)\right| Q, Q\right\rangle=R^{2} \sum_{m=0}^{Q} a_{m} \cos ^{2 m} \theta
$$

where $a_{m}$ are arbitrary (theory-dependent) constants subject to the constraint $\sum_{m} a_{m}=0$. By equating (27) to (28), we obtain the following predictions for $a_{m}$ at leading order:

$$
a_{m}= \begin{cases}\frac{\sqrt{Q}}{3 \alpha}, & \text { if } m=0 ; \\ 0, & \text { if } 1 \leq m \ll \sqrt{Q} .\end{cases}
$$

Because of the EFT cutoff (9), we can only make predictions for $m \ll \sqrt{Q}$. The constraint $\sum_{m} a_{m}=0$ is thus irrelevant for our discussion.
As a second example, we consider the states described by (3). Using (21), we find

$$
\left\langle\operatorname{vortex}\left|j_{\phi}(\theta, \phi)\right| \operatorname{vortex}\right\rangle=\frac{3 e^{2}}{8 \pi^{2} R} \frac{J}{Q} \sin ^{2} \theta .
$$

Rewriting (28) in the Fourier basis:

$$
\left\langle Q, Q\left|j_{\phi}(\theta, \phi)\right| Q, Q\right\rangle=R^{2} \sum_{m=0}^{J} b_{m} \cos 2 m \theta,
$$

we obtain the following predictions for $b_{m}$ at leading order:

$$
b_{m}= \begin{cases}\frac{(-1)^{m}}{8 \pi \alpha} \frac{J}{\sqrt{Q}}, & \text { if } m=0,1 ; \\ 0, & \text { if } 2 \leq m \ll \sqrt{J / Q} .\end{cases}
$$

Because we used a continuous approximation for the density $\rho$ (21), we can only make predictions for $m \ll$ $\sqrt{\rho} \sim \sqrt{J / Q}$.

\section{LARGE $N$}

So far, we assumed that the numerical coefficients in our effective Lagrangian are $O(1)$, corresponding to an underlying strongly coupled CFT. The case of a weakly coupled or large $N$ theory is quickly illustrated. However, the conclusions depend on whether the weak coupling appears in the $\chi$ description (4) or in the $a_{\mu}$ description (6). We will refer to the $\chi$ description as "electric" and to the $a_{\mu}$ one as "magnetic", with couplings $g_{e}^{2} \equiv 1 / N_{e}$ and $g_{m}^{2} \equiv 1 / N_{m}$, respectively.

Consider first a weakly coupled magnetic theory (for example, the large $N_{m}$ setup discussed in Refs. [44-51]). Since $1 / \alpha \sim g_{m}^{2}$ is small, the "bare" vortex mass $(\sim \gamma \sqrt{Q})$ is no longer subdominant to the electric field energy in (16). Therefore, the contribution $\frac{1}{2^{1 / 4}} n \gamma \sqrt{Q}$ should be added to (2) and (3), where $n=2$ for $J \leq Q$ and $n=3 J / Q$ for $J \gg Q$. For simplicity, we assumed the same bare mass for all vortices. This gives the dominant spin-dependent contribution to $\Delta$ for $J \lesssim Q / g_{m}^{2}$.

Consider now a weakly coupled electric theory. The essential difference in this case is that the cutoff is naturally identified with $\mu \sim g_{e} \sqrt{Q}$ [2] instead of with (9). Therefore, a single phonon is restricted to $J \lesssim g_{e} \sqrt{Q}$ and a vortexantivortex pair to $J \gtrsim Q \mu^{-1} \sim \sqrt{Q} / g_{e}$. States with $J$ in the gap between the two consist of multiple phonons, approaching a $1 / g_{e}^{2}$ number of them as $J \rightarrow \sqrt{Q} / g_{e}$. At this point, the lowest energy state shifts from multiple phonons to the vortex-antivortex pair. This consistently reflects the fact that vortices are now heavy solitons and consist of also roughly a $1 / g_{e}^{2}$ number of elementary quanta. Since $\alpha \sim g_{e}$, the logarithmic term in (2) is indeed the expected result, $\mu / g_{e}^{2} \ln \mu d$, for a semiclassical solution with a vortex-antivortex pair split by a distance $d=J / Q$. While 
possible a priori, we are not aware of any system in which the weakly coupled electric picture applies.

\section{HIGHER ORDER CORRECTIONS}

On general grounds, we expect corrections to come from higher derivative terms controlled by the cutoff length scale $l \equiv \Lambda^{-1}(9)$. Two classes of effects are expected. The first is controlled by the volume of the sphere and scales as $l^{2} / R^{2} \sim 1 / Q$. The second class is controlled by the separation $d$ among vortices and scales as $l^{2} / d^{2}$, where the double power of $d$ is dictated by rotational invariance. Using the relation $J \sim B d$ (20), we have $l^{2} / d^{2} \sim Q / J^{2}$.

In analyzing the possible terms in the Lagrangian, one indeed finds such corrections. The first class, already discussed in Refs. [1,3], arises from higher derivative corrections paired by conformal invariance with terms suppressed by $\mathcal{R} / f$, where $\mathcal{R}$ is the Riemann tensor. The second class arises from the mass term in (10). There we find relative corrections to the vortex action proportional to $\vec{E}^{2} / B^{2}$ and $\vec{X} \wedge \vec{E} / B$. These both scale as $l^{2} / d^{2} \sim Q / J^{2}$ on our solutions, becoming large at the lower edge $J \sim \sqrt{Q}$ of the two vortex states.

Notice that the second class of corrections is larger than the $\mathcal{O}(1 / J)$ quantum correction distinguishing (25) from the classical result (2). Nonetheless, the quantum correction is functionally distinguished and thus calculable. We also note that the universal $\mathcal{O}\left(Q^{0} J^{0}\right)$ contribution from the phonon Casimir energy [1,3] persists in the presence of vortices because the phonon spectrum is unmodified at leading order. We leave a systematic study of higher order corrections for future work.

\section{DISCUSSION}

To summarize, we calculated the scaling dimensions of operators with global charge $Q \gg 1$ and spin $J \lesssim Q^{3 / 2}$ by combining the state-operator correspondence with the EFT of vortices in superfluids. We also calculated correlation functions with two such operators and the Noether current. Other correlators as well as higher order corrections can be systematically computed.

Our results apply to any CFT whose large charge sector is described by the EFT we presented. To be clear, we have not proved that there actually exists any such CFT. However, what are the possibilities? Given a state with finite charge density, the $U(1)$ symmetry may or may not be spontaneously broken. If it is broken, then the state is a superfluid and our results generically apply. If it is not broken, then the state is not a superfluid (e.g., a Fermi liquid) and our results do not apply. Because superfluids are such a natural possibility, we believe that there exists a large class of CFTs to which our results apply.

Of course, it would be nice to explicitly identify such CFTs. One way forward is to consider CFTs that allow a perturbative expansion in some parameter and explicitly check if our results apply. For example, in $U(1)$ gauge theories, operators charged under the current (7) have been studied in a $1 / N$ expansion [44-51], where $N$ is the number of charged fields. The same operators were also studied in the $\epsilon$ expansion [52]. In both cases, results were only given for small $Q$, but the methods also apply at large $Q$. Related to large $N$, large charge states have also been studied via the AdS/CFT correspondence under the name of "holographic superconductors" [53-60].

Perhaps AdS/CFT can also teach us how to study operators with $J \sim \Delta$ using EFT techniques, as this was the original motivation for the large spin bootstrap work [13-23]. The idea was that these operators should be described as widely separated-and therefore weakly interacting-objects in AdS space [13]. This weak interaction suggests an EFT description, and such an EFT would then apply to all CFTs.

\section{ACKNOWLEDGMENTS}

We acknowledge useful discussions with João Penedones. The work of A. D., G. C., D. P. and R. R. is partially supported by the Swiss National Science Foundation under Contract No 200020-169696 and through the National Center of Competence in Research SwissMAP. The work of A. M. was supported by the ERCAdG-2015 Grant No. 694896 and the Swiss National Science Foundation (Ambizione). D. P. is supported by European Union's Horizon 2020 Research Council Grant No. 724659 MassiveCosmo ERC-2016-COG.
[1] S. Hellerman, D. Orlando, S. Reffert, and M. Watanabe, J. High Energy Phys. 12 (2015) 071.

[2] L. Alvarez-Gaume, O. Loukas, D. Orlando, and S. Reffert, J. High Energy Phys. 04 (2017) 059.

[3] A. Monin, D. Pirtskhalava, R. Rattazzi, and F. K. Seibold, J. High Energy Phys. 06 (2017) 011.
[4] O. Loukas, arXiv:1612.08985.

[5] S. Hellerman, N. Kobayashi, S. Maeda, and M. Watanabe, arXiv:1705.05825.

[6] S. Hellerman, S. Maeda, and M. Watanabe, arXiv:1706.05743.

[7] D. Banerjee, S. Chandrasekharan, and D. Orlando, arXiv: 1707.00711 . 
[8] O. Loukas, D. Orlando, and S. Reffert, arXiv:1707.00710.

[9] S. Hellerman and S. Maeda, arXiv:1710.07336.

[10] D. T. Son, arXiv:hep-ph/0204199.

[11] S. Rychkov, arXiv:1601.05000.

[12] D. Simmons-Duffin, in TASI 2015: New Frontiers in Fields and Strings (World Scientific, Singapore, 2016), Chap. 1, pp. $1-74$.

[13] A. L. Fitzpatrick, J. Kaplan, D. Poland, and D. SimmonsDuffin, J. High Energy Phys. 12 (2013) 004.

[14] Z. Komargodski and A. Zhiboedov, J. High Energy Phys. 11 (2013) 140.

[15] A. Kaviraj, K. Sen, and A. Sinha, J. High Energy Phys. 11 (2015) 083.

[16] L. F. Alday, A. Bissi, and T. Lukowski, J. High Energy Phys. 11 (2015) 101.

[17] A. Kaviraj, K. Sen, and A. Sinha, J. High Energy Phys. 07 (2015) 026.

[18] L. F. Alday and A. Zhiboedov, J. High Energy Phys. 06 (2016) 091.

[19] L. F. Alday and A. Zhiboedov, J. High Energy Phys. 04 (2017) 157.

[20] D. Li, D. Meltzer, and D. Poland, J. High Energy Phys. 02 (2016) 143.

[21] D. Simmons-Duffin, J. High Energy Phys. 03 (2017) 086.

[22] S. Caron-Huot, J. High Energy Phys. 09 (2017) 078.

[23] J. Qiao and S. Rychkov, arXiv:1709.00008.

[24] P. Dey, K. Ghosh, and A. Sinha, arXiv:1709.06110.

[25] R. Donnelly, Quantized Vortices in Helium II, Cambridge Studies in Low Temperature Physics No. 3 (Cambridge University Press, Cambridge, 1991).

[26] B. Horn, A. Nicolis, and R. Penco, J. High Energy Phys. 10 (2015) 153.

[27] R. Feynman, Statistical Mechanics: A Set Of Lectures, Advanced Books Classics (Avalon, New York, 1998).

[28] K. G. Wilson and M. E. Fisher, Phys. Rev. Lett. 28, 240 (1972).

[29] D. Jafferis, B. Mukhametzhanov, and A. Zhiboedov, arXiv: 1710.11161.

[30] L. Landau and E. Lifshitz, The Classical Theory of Fields, 4th ed., Course of Theoretical Physics (ButterworthHeinemann, London, 1975).

[31] L. Landau and E. Lifshitz, Quantum Mechanics, 3rd ed., Course of Theoretical Physics (Butterworth-Heinemann, London, 1977).

[32] N. Sivan and S. Levit, Phys. Rev. B 46, 2319 (1992).

[33] A. Entelis and S. Levit, Phys. Rev. Lett. 69, 3001 (1992).

[34] T. Tochishita, M. Mizui, and H. Kuratsuji, Phys. Lett. A 212, 304 (1996).
[35] G. V. Dunne, R. Jackiw, and C. A. Trugenberger, Phys. Rev. D 41, 661 (1990).

[36] G. Dunne and R. Jackiw, Nucl. Phys. B, Proc. Suppl. 33, 114 (1993).

[37] T. T. Wu and C. N. Yang, Nucl. Phys. B107, 365 (1976).

[38] S. Coleman, in The Unity of the Fundamental Interactions, edited by A. Zichichi (Springer, New York, 1983), pp. 21-117.

[39] M. Hatsuda, S. Iso, and H. Umetsu, Nucl. Phys. B671, 217 (2003).

[40] K. Hasebe, SIGMA 6, 071 (2010).

[41] F. D. M. Haldane, Phys. Rev. Lett. 51, 605 (1983).

[42] M. Greiter, Phys. Rev. B 83, 115129 (2011).

[43] M. S. Costa, J. Penedones, D. Poland, and S. Rychkov, J. High Energy Phys. 11 (2011) 071.

[44] G. Murthy and S. Sachdev, Nucl. Phys. B344, 557 (1990).

[45] V. Borokhov, A. Kapustin, and X.-k. Wu, J. High Energy Phys. 11 (2002) 049.

[46] M. A. Metlitski, M. Hermele, T. Senthil, and M. P. A. Fisher, Phys. Rev. B 78, 214418 (2008).

[47] S. S. Pufu and S. Sachdev, J. High Energy Phys. 09 (2013) 127.

[48] S. S. Pufu, Phys. Rev. D 89, 065016 (2014).

[49] E. Dyer, M. Mezei, and S. S. Pufu, arXiv:1309.1160.

[50] E. Dyer, M. Mezei, S. S. Pufu, and S. Sachdev, J. High Energy Phys. 06 (2015) 037; 03 (2016) 111(E).

[51] S. M. Chester, L. V. Iliesiu, M. Mezei, and S. S. Pufu, arXiv:1710.00654.

[52] S. M. Chester, M. Mezei, S. S. Pufu, and I. Yaakov, J. High Energy Phys. 12 (2016) 015.

[53] S. A. Hartnoll, C. P. Herzog, and G. T. Horowitz, Phys. Rev. Lett. 101, 031601 (2008).

[54] S. A. Hartnoll, C. P. Herzog, and G. T. Horowitz, J. High Energy Phys. 12 (2008) 015.

[55] S. A. Hartnoll, Classical Quantum Gravity 26, 224002 (2009).

[56] C. P. Herzog, J. Phys. A 42, 343001 (2009).

[57] G. T. Horowitz and M. M. Roberts, J. High Energy Phys. 11 (2009) 015.

[58] G. T. Horowitz, in From Gravity to Thermal Gauge Theories: The AdS/CFT Correspondence, Lect. Notes Phys. Vol. 828, edited by E. Papantonopoulos (Springer, Berlin, 2011), pp. 313-347.

[59] S. A. Hartnoll, arXiv:1106.4324.

[60] Ó. J. C. Dias, G. T. Horowitz, N. Iqbal, and J. E. Santos, J. High Energy Phys. 04 (2014) 096. 